\title{
$\epsilon$-CONVERTIBILITY OF ENTANGLED STATES AND EXTENSION OF SCHMIDT RANK IN INFINITE-DIMENSIONAL SYSTEMS
}

\author{
Masaki Owari \\ Collaborative Institute for Nano Quantum Information Electronics, The University of Toky ${ }^{a}$ \\ Tokyo 113-0033, Japan \\ Samuel L. Braunstein \\ Computer Science, University of York \\ York YO10 5DD, United Kingdom \\ Kae Nemoto \\ National Institute of Informatics \\ Tokyo 101-8430, Japan \\ Mio Murao \\ Department of Physics, The University of Tokyo \\ Tokyo 113-0033, Japan \\ PRESTO, JST \\ Kawaguchi, Saitama 332-0012, Japan
}

\begin{abstract}
By introducing the concept of $\epsilon$-convertibility, we extend Nielsen's and Vidal's theorems to the entanglement transformation of infinite-dimensional systems. Using an infinitedimensional version of Vidal's theorem we derive a new stochastic-LOCC (SLOCC) monotone which can be considered as an extension of the Schmidt rank. We show that states with polynomially-damped Schmidt coefficients belong to a higher rank of entanglement class in terms of SLOCC convertibility. For the case of Hilbert spaces of countable, but infinite dimensionality, we show that there are actually an uncountable number of classes of pure non-interconvertible bipartite entangled states.
\end{abstract}

Keywords: Entanglement, LOCC, Infinite dimension, Continuous variable

\section{Introduction}

Entanglement is one of the central topics in quantum information, and has both physical and information scientific aspects. In particular, entanglement involves quantum non-local correlations which have been of interest in physics [1, and also acts as a resource of quantum information processing for informatics 2, 3. Thus, the characterization of the entanglement

${ }^{a}$ The major part of this work was done when MO was in Department of Physics, Graduate School of Science, The University of Tokyo. 
of physical systems is important from both a physical and information scientific viewpoint.

Mathematically, physical systems can be categorized into two classes, that is, finitedimensional systems which can be treated in the framework of conventional linear algebra, and infinite-dimensional systems which need to be treated in the framework of functional analysis [4, 5. It is therefore worthwhile to know whether such a mathematical difference of systems can make an essential difference in the properties of entanglement in these systems. Indeed, this may provide an answer to the question of what is the essential difference between the physics of finite-dimensional systems and the physics of infinite-dimensional systems from the viewpoint of non-local correlations. Moreover, from the information-theoretic viewpoint, if such a difference exists, there may be the possibility that we can achieve an information processing in infinite-dimensional systems which cannot be achieved in finite-dimensional systems.

In this paper, we mainly focus on seeking a difference between the properties of entanglement of finite-dimensional systems and those of infinite-dimensional systems. Since much work on the characterization of bipartite entangled states has been done in finite-dimensional systems [6, 7, 8, 9, we concentrate our efforts on the characterization of bipartite entangled states in infinite-dimensional systems, and try to find a difference in the properties of their entanglement.

So far, research on the characterization of entanglement in infinite dimensions has been done in the form of separability criteria [10, Gaussian LOCC convertibility [11, 12, and entanglement measures [13. The separability criteria gives us a way of judging whether or not a given state is entangled. The Gaussian LOCC convertibility gives the detailed structure of the strength of entanglement of Gaussian states. Entanglement measures give an approximated strength of entanglement in the limit of an asymptotic infinite number of copies.

The above research mainly is concerned with Gaussian states and Gaussian operations, and unique properties of infinite-dimensional entanglement do not appear clearly in this regime. Therefore, in order to find a property unique to infinite-dimensional entanglement it is important to investigate the strength of entanglement more precisely for a broader class of states and operations.

The strength of entanglement is defined by means of the convertibility between entangled states under local operations, e.g., local operations and classical communication (LOCC), stochastic-LOCC (SLOCC), or the positive partial transpose (PPT) operation 14, 15. Among such local operations we mainly focus in this paper on SLOCC, and investigate the SLOCC convertibility of entangled states in infinite-dimensional systems without any assumption for states or operations to find a unique property of entanglement in infinite-dimensional systems.

When we consider SLOCC convertibility for infinite-dimensional systems, there are at least two difficulties, namely, the problem of continuity and the problem of a potentially infinite cost for classical communication. In order to avoid such difficulties, we propose a new definition of state convertibility that we call $\epsilon$-convertibility. We define $\epsilon$-convertibility as the convertibility of states in an approximated setting by means of the trace norm. Then, within the framework of $\epsilon$-convertibility, we investigate SLOCC convertibility in infinite-dimensional systems and show a fundamental difference of SLOCC convertibility between infinite and finite-dimensional systems. 
The paper is organized as follows: in section 2 we define the $\epsilon$-convertibility of LOCC and SLOCC, and show how to avoid the problems of discontinuity and infinitely-costly classical communication. Then within the framework of $\epsilon$-convertibility, we give the infinitedimensional extensions and proofs of Nielsen's and Vidal's theorem, which give the necessary and sufficient conditions of LOCC and SLOCC convertibility, respectively. In section 3, first we define monotones (monotonic functions) of SLOCC convertibility, which can be considered an extension of the Schmidt rank for infinite-dimensional systems, then by means of this monotone, we investigate the SLOCC convertibility for infinite-dimensional systems. We show that the cardinal number of the quotient set of states by SLOCC convertibility is greater than or equal to the cardinal number of the continuum, and also show that however many (finite) copies of exponentially-damped states (states with exponentially damped Schmidt coefficients) there are, they cannot be converted into even a single copy of a polynomiallydamped state (a state with polynomially-damped Schmidt coefficients). Such properties do not exist in finite-dimensional systems and are actually unique to infinite-dimensional systems.

\section{$2 \epsilon$-convertibility}

In this paper we consider the bipartite infinite-dimensional system $\mathcal{H}=\mathcal{H}_{A} \otimes \mathcal{H}_{B}$ where $\operatorname{dim} \mathcal{H}_{A}=\operatorname{dim} \mathcal{H}_{B}=\infty$ and we shall assume that $\mathcal{H}_{A}$ and $\mathcal{H}_{B}$ are separable. By $\mathfrak{B}(\mathcal{H})$ we denote the Banach space of all bounded operators on $\mathcal{H}$. If we use the term LOCC, we will always assume that operations succeed with unit probability [15]. On the other hand we use the term SLOCC in the case where operations work with a finite probability less than unity. For simplicity we use at most countably infinite POVMs as the element of an LOCC (or SLOCC), $\left\{A_{i}\right\}_{i=1}^{\infty}, A_{i} \in \mathfrak{B}(\mathcal{H}), \sum_{i \in \mathbb{N}} A^{\dagger} A=($ or $\leq) I$ (corresponding to ultra-weak convergence).

\section{$2.1 \epsilon$-convertibility for LOCC and SLOCC}

As mentioned in the introduction to give a detailed discussion of SLOCC convertibility in infinite-dimensional systems, there are at least two difficulties, namely, discontinuity and infinite classical communication costs. In this subsection we define $\epsilon$-convertibility and see that it allows us to avoid the difficulty of discontinuity. The other difficulty is addressed in the following subsection.

In infinite-dimensional systems we cannot deny the possibility that $|\Psi\rangle$ is SLOCC convertible to any neighborhood of $|\Phi\rangle$ (in terms of strong, or weak topology), but not to $|\Phi\rangle$ itself. To avoid such a discontinuity, when considering convertibility among genuinely infinitedimensional states (i.e., states with infinitely many non-zero Schmidt coefficients), we shall identify these neighborhoods with the state itself. To achieve this we shall extend the definition of LOCC and SLOCC convertibility to satisfy the above requirement. Mathematically, we redefine LOCC convertibility as follows: $|\Psi\rangle$ can be converted to $|\Phi\rangle$ by LOCC, if and only if for any neighborhood of $|\Phi\rangle$, there exists an LOCC operation by which $|\Psi\rangle$ is transformed to a state in the neighborhood of $|\Phi\rangle$. We call this new definition of convertibility $\epsilon$-convertibility. Below we rigorously define $\epsilon$-convertibility for LOCC, then we show that this definition recovers the continuity of convertible probability at least with some suitable weak meaning.

Before we give the definition of $\epsilon$-convertibility, we need to choose a topology of the convergence which we use in our definition. Actually, it is well known that there are many 
different topologies defined by associated norms in infinite-dimensional systems. Therefore, we need to take care to choose our 'distance'. Since we introduced $\epsilon$-convertibility because of the fundamental impossibility for discriminating a state $|\Phi\rangle$ from states within infinitely small neighborhoods of $|\Phi\rangle$, the distance we consider needs to echo this difficulty with discrimination. We can easily see that the trace norm possesses such a property as follows. Suppose $M$ is an arbitrary POVM element and $\lim _{n \rightarrow \infty}\left\|\rho-\rho_{n}\right\|_{\text {tr }}=0$. Then, $\lim _{n \rightarrow \infty}\left|\operatorname{tr} \rho M-\operatorname{tr} \rho_{n} M\right| \leq \lim _{n \rightarrow \infty}\left\|\rho-\rho_{n}\right\|_{\text {tr }}\|M\|_{\text {op }}=0$, where $\|\cdot\|_{\text {op }}$ is the operator norm. Thus, for all measurements the resulting probability distributions for $\rho_{n}$ converge to the resulting probability distribution for $\rho$. That is, if $\rho_{n}$ converges $\rho$ in the trace norm, there is no way to discriminate $\rho$ from $\rho_{n}$ for sufficiently large $n$. But this is just the property required of the distance needed to deal with the discontinuity difficulty in the definition of $\epsilon$-convertibility. Therefore, we shall use the trace norm as our distance measure for $\epsilon$-convertibility.

Following this discussion we rigorously define $\epsilon$-convertibility for LOCC as:

Definition 1 We say that $|\Psi\rangle$ is $\epsilon$-convertible to $|\Phi\rangle$ by LOCC, if for any $\epsilon>0$, there exists an LOCC operation $\Lambda$ which satisfies the condition $\| \Lambda(|\Psi\rangle\langle\Psi|)-|\Phi\rangle\langle\Phi| \|_{\text {tr }}<\epsilon$ where $\|\cdot\|_{\text {tr }}$ is the trace norm.

Similarly, we define $\epsilon$-convertibility for SLOCC as:

Definition 2 We say that $|\Psi\rangle$ is $\epsilon$-convertible to $|\Phi\rangle$ by SLOCC with probability $p>0$ if for any $\epsilon>0$, there exists an SLOCC operation $\Lambda$ which satisfies the following condition, $\| \Lambda(|\Psi\rangle\langle\Psi|) / \operatorname{tr} \Lambda(|\Psi\rangle\langle\Psi|)-|\Phi\rangle\langle\Phi| \|_{\text {tr }}<\epsilon$ and $\operatorname{tr} \Lambda(|\Psi\rangle\langle\Psi|) \geq p$.

This definition of $\epsilon$-convertibility under SLOCC means that with more than some fixed nonzero probability $p,|\Psi\rangle$ can be converted to any neighborhood of $|\Phi\rangle$ by SLOCC.

Here, we prove by means of $\epsilon$-convertibility that we can recover enough continuity to achieve a classification of states by SLOCC convertibility. In infinite-dimensional systems when we consider SLOCC convertibility it might happen that $|\Psi\rangle$ cannot be converted to $|\Phi\rangle$ and yet there exists a sequence of $\left|\Phi_{n}\right\rangle$ such that $|\Psi\rangle$ can be converted to $\left|\Phi_{n}\right\rangle$ with probability $p_{n}$ and $\lim _{n \rightarrow \infty} p_{n}>0$; however, we cannot discriminate $|\Phi\rangle$ and $\left|\Phi_{n}\right\rangle$ for large $n$. If this were to happen then it would be nonsense that $|\Psi\rangle$ could not converted to $|\Phi\rangle$ by SLOCC. ${ }^{a}$ However, by means of our new definition of convertibility, we avoid such a discontinuity. That is, we can easily show the following continuity property of $\epsilon$-convertibility.

Lemma 1 If $|\Psi\rangle$ is not $\epsilon$-convertible to $|\Phi\rangle$ by SLOCC, but $|\Psi\rangle$ is $\epsilon$-convertible to $\left|\Phi_{n}\right\rangle$ by SLOCC with probability $p_{n}$ for all $n$, where $\lim _{n \rightarrow \infty}\left|\Phi_{n}\right\rangle\left\langle\Phi_{n}|=| \Phi\right\rangle\langle\Phi|$ by the trace norm, then $\lim _{n \rightarrow \infty} p_{n}=0$.

Proof We prove this lemma by contradiction. We assume the following condition; $|\Psi\rangle$ is not $\epsilon$-convertible to $|\Phi\rangle,|\Psi\rangle$ is $\epsilon$-convertible to $\left|\Phi_{n}\right\rangle$ with probability $p_{n}>0$, where $\lim _{n \rightarrow \infty}\left|\Phi_{n}\right\rangle=|\Phi\rangle$. Moreover, if we add one condition; $\lim _{\sup _{n \rightarrow \infty}} p_{n}>0$, then we can show the contradiction as follows.

\footnotetext{
${ }^{a}$ To avoid confusion, we add a remark. We do not know an explicit example of this discontinuity. However, in infinite dimensional systems, it is not trivial whether such discontinuity occurs or not. Hence, it is better to introduce a modified definition of convertibility under which we can trivially avoid the discontinuity.
} 
Since $\lim \sup _{n \rightarrow \infty} p_{n}>0$, there exists a subsequence of $p_{n}$, such that $\lim p_{n(k)}=p>0$ and $p_{n(k)}>p / 2$ for all $k \in \mathbb{N}$. Then, since $|\Psi\rangle$ is $\epsilon$-convertible to $\left|\Phi_{n}\right\rangle$ with probability $p_{n}$, for any $\epsilon>0$ and for any $k \in \mathbb{N}$, there exists an SLOCC operation $\Lambda_{\epsilon, n(k)}$ such that $\| \Lambda_{\epsilon, n(k)}(|\Psi\rangle\langle\Psi|) / \operatorname{tr} \Lambda_{\epsilon, n(k)}(|\Psi\rangle\langle\Psi|)-\left|\Phi_{n(k)}\right\rangle\left\langle\Phi_{n(k)}\right| \|<\epsilon$ and $\left.\operatorname{tr} \Lambda_{\epsilon, n(k)}(|\Psi\rangle\langle\Psi|) \geq p_{n(k)}\right\rangle$ $p / 2$. Moreover, since $\lim _{n \rightarrow \infty}\left|\Phi_{n}\right\rangle=|\Phi\rangle$, for any $\epsilon>0$, there exists an $N_{\epsilon} \in \mathbb{N}$ such that for any $n \geq N_{\epsilon}, \|\left|\Phi_{n}\right\rangle\left\langle\Phi_{n}|-| \Phi\right\rangle\langle\Phi| \|<\epsilon$. Therefore, for any $2 \epsilon>0$, by choosing $k \in \mathbb{N}$ as $n(k) \geq N_{\epsilon}$,

$$
\begin{aligned}
& \| \Lambda_{\epsilon, n(k)} / \operatorname{tr} \Lambda_{\epsilon, n(k)}-|\Phi\rangle\langle\Phi| \| \\
\leq & \| \Lambda_{\epsilon, n(k)} / \operatorname{tr} \Lambda_{\epsilon, n(k)}-\left|\Phi_{n(k)}\right\rangle\left\langle\Phi_{n(k)}|\|+\|| \Phi_{n(k)}\right\rangle\left\langle\Phi_{n(k)}|-| \Phi\right\rangle\langle\Phi| \| \\
\leq & 2 \epsilon .
\end{aligned}
$$

Moreover, $\operatorname{tr} \Lambda_{\epsilon, n(k)}(|\Psi\rangle\langle\Psi|) \geq p_{n(k)}>p / 2$. Therefore, $|\Psi\rangle$ is $\epsilon$-convertible to $|\Phi\rangle$ with probability $p / 2$. This is a contradiction. Therefore, if $|\Psi\rangle$ is not $\epsilon$-convertible to $|\Phi\rangle$, and if $|\Psi\rangle$ is $\epsilon$-convertible to $\left|\Phi_{n}\right\rangle$ with probability $p_{n}$ where $\lim _{n \rightarrow \infty}\left|\Phi_{n}\right\rangle=|\Phi\rangle$, then, $\lim _{n \rightarrow \infty} p_{n}=0$. square

This lemma means that if $|\Psi\rangle$ cannot be converted to $|\Phi\rangle$ by SLOCC, then $|\Psi\rangle$ is also almost certainly inconvertible to states near to $|\Phi\rangle$. Therefore, our definition of $\epsilon$-convertibility preserves continuity of the theory (at least sufficiently for the purposes of the classification of states), and we can avoid the discontinuity difficulty mentioned above.

\subsection{Nielsen's and Vidal's theorems for infinite-dimensional systems}

In this subsection we reconstruct Nielsen's and Vidal's theorems for infinite-dimensional systems by means of $\epsilon$-convertibility. As a result, we will see that we can also avoid the difficulty of a potentially infinite cost for classical communication by our convertibility, that is, only a finite amount of classical communication is actually necessary for our theory of convertibility. As is well known, Nielsen's and Vidal's theorems give the necessary and sufficient conditions of LOCC and SLOCC, respectively. Therefore, by proving these theorems rigorously we may obtain a firm foundation for the analysis of SLOCC convertibility for infinite-dimensional systems, which we shall consider in the next section. Since Vidal's theorem is a generalization of Nielsen's theorem, we shall first discuss Nielsen's theorem and then go on to consider Vidal's theorem.

In finite-dimensional systems Nielsen's theorem gives the necessary and sufficient conditions for LOCC convertibility between a pair of bipartite pure states $|\Phi\rangle$ and $|\Psi\rangle$ as follows

$$
|\Psi\rangle \rightarrow|\Phi\rangle \quad \Leftrightarrow \quad \lambda \prec \mu
$$

where the arrow $\rightarrow$ represents convertibility under LOCC, and $\lambda$ and $\mu$ represent sequences of Schmidt coefficients (in descending order) of the states $|\Psi\rangle$ and $|\Phi\rangle$, respectively, and $\prec$ denotes majorization of the sequences [7] (if $\lambda \prec \mu$, we say " $\lambda$ is majorized by $\mu$ "). In infinitedimensional systems we can show that Eq. (11) is still valid where we replace the meaning of $\rightarrow$ by $\epsilon$-convertibility under LOCC. Nielsen's theorem then takes the following form for infinite-dimensional systems: 
Theorem $1|\Psi\rangle$ is $\epsilon$-convertible to $|\Phi\rangle$, if and only if $\lambda \prec \mu$, where $\prec$ means majorization in infinite-dimensional systems (see Appendix (B), and $\lambda$ and $\mu$ are the Schmidt coefficients of $|\Psi\rangle$ and $|\Phi\rangle$, respectively.

Since the proof of theorem 1 is long, we have placed the rigorous proof of this theorem in Appendix [C] Below we only give a sketch of the proof:

\section{Sketch of Proof}

1) The necessary part: We can directly extend the proof of necessity of the original theorem to infinite-dimensional systems. The necessary condition part of the original theorem is constructed using the Lo-Popescu theorem (Theorem [5] [16] and Uhlmann's theorem (Theorem (6). Since these two theorems can themselves be extended to infinite-dimensional systems (see Appendix A and B). The same proof for finite-dimensional systems still holds in infinitedimensional systems.

2) The sufficient part: In the proof of sufficiency, our definition of $\epsilon$-convertibility plays a crucial role in extending the proof of Nielsen's theorem. Our proof is based on the proof for finite-dimensional systems in Ref. [15] and is extended to genuine infinite-dimensional states by means of $\epsilon$-convertibility. We can show that for any $N$, there exists a state $\left|\Phi^{\prime}\right\rangle$ (which depends on $N$ ) such that its first $N$ Schmidt coefficients are equal to the Schmidt coefficients of $|\Phi\rangle$ and where the Schmidt coefficients of $|\Psi\rangle$ are majorized by the Schmidt coefficients of $\left|\Phi^{\prime}\right\rangle$. Therefore, for every neighborhood of $|\Phi\rangle$, we can always find a state to which $|\Psi\rangle$ can be converted under LOCC.

By means of Nielsen's theorem in infinite-dimensional systems we can extend Vidal's theorem for SLOCC convertibility [ $[$, which gives the necessary and sufficient condition of SLOCC convertibility with a probability $p$ to infinite-dimensional systems using $\epsilon$-convertibility. Vidal's theorem states that a bipartite pure state $|\Psi\rangle$ can be converted to another bipartite pure state $|\Phi\rangle$ under SLOCC with probability at least $p$ if and only if $\lambda \prec^{\omega} p \mu$ [here $\prec^{\omega}$ denotes super-majorization and is defined in Appendix A1, Eq. (B.3) of Definition 4]. The generalization of Vidal's theorem for $\epsilon$-convertibility can then be written:

Theorem $2|\Psi\rangle$ is $\epsilon$-convertible to $|\Phi\rangle$ by SLOCC with probability $p$, if and only if $\lambda \prec^{\omega} p \mu$ are satisfied where $\lambda$ and $\mu$ are the Schmidt coefficients of $|\Psi\rangle$ and $|\Phi\rangle$ respectively.

Proof The proof of this theorem is in appendix D.

Therefore, the extension of Vidal's theorem also applies to $\epsilon$-convertibility.

Although infinite amounts of classical information do not exist in the real world, an infinite amount of classical communication is necessary to convert one genuine infinite-dimensional state to another by LOCC and SLOCC in the conventional theory of convertibility. From the proof of Theorem 1, we can show that we can avoid such infinite costs of classical communication in LOCC convertibility by our new definition of $\epsilon$-convertibility. In the proof of this theorem, we showed that there exists a natural number $M$ such that $\left|\Phi^{\prime}\right\rangle$ satisfies the condition $\mu_{N}^{\prime}=\lambda_{N}$ for $N \geq M$. Therefore, the LOCC operation by which $|\Psi\rangle$ can be converted into $\left|\Phi^{\prime}\right\rangle$ is actually an LOCC operation requiring only a finite amount of classical communication. We can also show a similar result for SLOCC convertibility. By the proof of Theorem 2 it is easily seen that we can construct the protocol of SLOCC with only a finite amount 
of classical communication in a manner similar to LOCC. As a result, in our definition of $\epsilon$-convertibility of LOCC and SLOCC, we can convert states with any finite accuracy by only a finite amount of communication, and only when this error goes to zero does the amount of classical communication go to infinity. Therefore, our definition of $\epsilon$-convertibility yields a theory of single-copy LOCC and SLOCC convertibility requiring only a finite amount of classical communication even in the infinite-dimensional setting.

Here we need to add two final remarks about our framework of $\epsilon$-convertibility. From the proofs of Theorems 1 and 2, we can derive another interpretation of $\epsilon$-convertibility. First, in the case of LOCC, that is, Nielsen's theorem, since the state $\left|\Phi^{\prime}\right\rangle=\sum_{k=1}^{\infty} \sqrt{\mu^{\prime}}|i\rangle \otimes|i\rangle$ is also majorized by $|\Phi\rangle$ in the proof of Theorem 1 (Appendix [C), we can immediately see the following fact: If $\lambda \prec \mu$ where $\lambda$ and $\mu$ are the Schmidt coefficients of $|\Phi\rangle$ and $|\Psi\rangle$ respectively, then there exists a sequence of LOCC $\left\{\Lambda_{n}\right\}_{n=1}^{\infty}$ such that for all $n \in \mathbb{N}, \Lambda_{n} \cdots \Lambda_{1}(|\Psi\rangle\langle\Psi|)$ has the same Schmidt basis and their Schmidt coefficients are majorized by those of $|\Phi\rangle$ and they also satisfy $\lim _{n \rightarrow \infty} \Lambda_{n} \cdots \Lambda_{1}(|\Psi\rangle\langle\Psi|)=|\Phi\rangle\langle\Phi|$. Thus, we can interpret the above sequence of LOCC as the LOCC with an infinite number of steps of classical communication. Second, since in the proof of the above theorem in appendix $\mathrm{D}$, we constructed a sequence of SLOCC $\left\{\Lambda_{n}\right\}_{n=1}^{\infty}$ such that $\lim _{n \rightarrow \infty} \Lambda_{n} \cdots \Lambda_{1}(|\Psi\rangle\langle\Psi|) / \operatorname{tr} \Lambda_{n} \cdots \Lambda_{1}(|\Psi\rangle\langle\Psi|)=|\Phi\rangle\langle\Phi|$, we can consider that Vidal's theorem is also naturally extended to infinite-dimensional systems by the redefinition of LOCC convertibility including an infinite number of steps of classical communication. Therefore, we can also say that both the Nielsen and Vidal theorems can be extended to infinite-dimensional systems if we allow for an infinite number of steps of LOCC.

In this section, we proposed a new definition of convertibility, $\epsilon$-convertibility, to treat entanglement convertibility between genuine infinite-dimensional states. This redefinition is suitable from both the technical and realistic viewpoints, that is, to avoid the difficulties of both discontinuity and infinite cost in classical communication for infinite-dimensional systems. Then, by means of $\epsilon$-convertibility we proved the Nielsen and Vidal theorems which are the fundamental theorems of LOCC and SLOCC convertibility in infinite-dimensional systems. As a result under our change of definition the framework of entanglement convertibility is preserved in the context of infinite-dimensional systems, and therefore our definition of $\epsilon$-convertibility for LOCC is suitable and sufficient for realistic conditions of quantum information processing in infinite-dimensional systems.

\section{Extension of Schmidt rank}

\subsection{Definition and its basic property}

In this section we discuss the SLOCC convertibility of infinite-dimensional systems and show that there are many important differences between the structure of the SLOCC classification of genuinely infinite-dimensional states and that of finite-dimensional states. For this purpose in this subsection we first define a pair of new SLOCC monotones which can be considered as extensions of the Schmidt rank, and then we analyze their properties. Finally, we show that there are continuously many classes of states under SLOCC convertibility in infinite-dimensional systems. In the following we always consider SLOCC convertibility in the meaning of $\epsilon$-convertibility defined above. Therefore, we henceforth omit the qualifier ' $\epsilon$ '.

To study convertibility in detail, monotones of convertibility are crucially important. In finite systems, the Schmidt rank (the rank of the reduced density matrix) gives the necessary 
and sufficient condition of SLOCC convertibility. On the other hand, in infinite-dimensional systems, since almost all states have infinite Schmidt rank, the classification by Schmidt rank is not useful. Therefore, proposals for new SLOCC monotones for genuine infinite-dimensional entangled states are essential for analysis of SLOCC convertibility between genuinely infinitedimensional states. In the followings, we give a definition of a pair of new SLOCC monotones $R^{-}$and $R^{+}$, which can be considered an extension of the Schmidt rank for infinite-dimensional systems.

Since the usual Schmidt rank represents how quickly Schmidt coefficients vanish, when we consider their extension to genuine infinite-dimensional states it is natural to define the extension of this concept as a function which represents how quickly a sequence of Schmidt coefficients converge to zero. In Vidal's theorem Schmidt coefficients always appear in the form of a sum from $n$ to $\infty$ which is an LOCC monotone for all $n \in \mathbb{N}$ and is called "Vidal's monotone" 9]. Therefore, rather than studying the direct convergence of Schmidt coefficients $\left\{\lambda_{n}\right\}_{n=1}^{\infty}$ we shall study the convergence of Vidal's monotones $\left\{\sum_{i=n}^{\infty} \lambda_{i}\right\}_{n=1}^{\infty}$. To measure the speed of convergence of Vidal's monotone we compare a sequence of Vidal's monotones with some real parameterized class of sequences. Thus, we define the new monotones as follows:

Definition 3 For a parameterized class of sequences $\left\{f_{r}(n)\right\}_{r \in(a, b)}$ which satisfy the following conditions:

$$
\begin{aligned}
& \forall r \in(a, b), \lim _{n \rightarrow \infty} f_{r}(n)=0 \\
& \forall r \in(a, b), n_{1}<n_{2} \Rightarrow f_{r}\left(n_{1}\right)>f_{r}\left(n_{2}\right)>0 \\
& \forall n \in \mathbb{N}, r_{1}<r_{2} \Rightarrow \lim _{n \rightarrow \infty} \frac{f_{r_{1}}(n)}{f_{r_{2}}(n)}=0,
\end{aligned}
$$

where $n \in \mathbb{N}, 0 \leq a<b \leq \infty$, we define a pair of functions $R_{f_{r}}^{+}(|\Psi\rangle)$ and $R_{f_{r}}^{-}(|\Psi\rangle)$ by

$$
\begin{aligned}
& R_{f_{r}}^{+}(|\Psi\rangle)=\inf \left\{r \in(a, b) \mid \lim _{n \rightarrow \infty} \frac{\sum_{i=n}^{\infty} \lambda_{i}}{f_{r}(n)}=0\right\} \\
& R_{f_{r}}^{-}(|\Psi\rangle)=\inf \left\{r \in(a, b) \varliminf_{n \rightarrow \infty} \frac{\sum_{i=n}^{\infty} \lambda_{i}}{f_{r}(n)}=0\right\} .
\end{aligned}
$$

If for all $r \in(a, b), \varlimsup_{n \rightarrow \infty} \sum_{i=n}^{\infty} \lambda_{i} / f_{r}(n)>0$, then we define $R_{f_{r}}^{+}(|\Psi\rangle)=b$. Here, we use the notation of $\varlimsup=\lim \sup$ and $\underline{\lim }=\lim$ inf.

For the definition of $R_{f_{r}}^{+}(|\Psi\rangle)$, we could also have defined this function as

$$
R_{f_{r}}^{+}(|\Psi\rangle)=\inf \left\{r \in(a, b) \mid \overline{\lim }_{n \rightarrow \infty} \frac{\sum_{i=n}^{\infty} \lambda_{i}}{f_{r}(n)}=0\right\},
$$

however this definition is the same as the previous definition, since $\varlimsup_{n \rightarrow \infty} \sum_{i=n}^{\infty} \lambda_{i} / f_{r}(n)=0$ guarantees $\lim _{n \rightarrow \infty} \sum_{i=n}^{\infty} \lambda_{i} / f_{r}(n)=0$. Note that the limits $\overline{\lim }_{n \rightarrow \infty} \sum_{i=n}^{\infty} \lambda_{i} / f_{r}(n)$ and $\underline{\lim }_{n \rightarrow \infty} \sum_{i=n}^{\infty} \lambda_{i} / f_{r}(n)$ do not generally coincide. Thus, to measure the speed of the convergence, we need two functions $R_{f_{r}}^{+}(|\Psi\rangle)$ and $R_{f_{r}}^{-}(|\Psi\rangle)$ corresponding to these different approaches of the limit as given above. By their definition, we can easily see that $R_{f_{r}}^{+}$and $R_{f_{r}}^{-}$ satisfy $R_{f_{r}}^{-}(|\Psi\rangle) \leq R_{f_{r}}^{+}(|\Psi\rangle)$ for all $|\Psi\rangle$. As we might expect, both $R_{f_{r}}^{+}$and $R_{f_{r}}^{-}$are SLOCC 
monotones, and moreover, the sufficient condition of monotonicity is also partially valid as given by the statement of the following theorem. (Note, that when the choice of $f_{r}(x)$ is clear we write simply $R^{+}$and $R^{-}$for the monotones.)

Theorem 3 For all $f_{r}$ which satisfy the condition in Definition 3

1. If $|\Psi\rangle$ can be converted to $|\Phi\rangle$ by SLOCC then $R_{f_{r}}^{+}(|\Psi\rangle) \geq R_{f_{r}}^{+}(|\Phi\rangle)$ and $R_{f_{r}}^{-}(|\Psi\rangle) \geq$ $R_{f_{r}}^{-}(|\Phi\rangle)$.

2. If $R_{f_{r}}^{+}(|\Phi\rangle)<R_{f_{r}}^{-}(|\Psi\rangle)$, then $|\Psi\rangle$ can be converted to $|\Phi\rangle$ by SLOCC.

Proof Proof of part 1:

We only prove this for the case of $R_{f_{r}}^{+}$since the proof for $R_{f_{r}}^{-}$is identical. Suppose $R_{f_{r}}^{+}(|\Phi\rangle)>$ $R_{f_{r}}^{+}(|\Psi\rangle)$ then for all $R_{f_{r}}^{+}(|\Psi\rangle)<r<R_{f_{r}}^{+}(|\Phi\rangle), \varlimsup_{n \rightarrow \infty} \sum_{i=n}^{\infty} \lambda_{i} / f_{r}(n)=0$ and

$\varlimsup_{n \rightarrow \infty} \sum_{i=n}^{\infty} \mu_{i} / f_{r}(n)>0$, where $\left\{\lambda_{i}\right\}_{i=0}^{\infty}$ and $\left\{\mu_{i}\right\}_{i=0}^{\infty}$ are Schmidt coefficients of $|\Psi\rangle$ and $|\Phi\rangle$, respectively. Thus, for all $\delta>0$ there exists an $N_{0}(\delta)$ such that if $n>N_{0}(\delta)$, then $\sum_{i=n}^{\infty} \lambda_{i} / f_{r}(n)<\delta$. Suppose $a \stackrel{\text { def }}{=} \varlimsup_{n \rightarrow \infty} \sum_{i=n}^{\infty} \mu_{i} / f_{r}(n)>0$, then there exists a partial sequence of $\sum_{i=n}^{\infty} \mu_{i} / f_{r}(n)$, say $\sum_{i=k(n)}^{\infty} \mu_{i} / f_{r}(k(n))$, such that $\lim _{n \rightarrow \infty} \sum_{i=k(n)}^{\infty} \mu_{i} / f_{r}(k(n))=$ $a>0$. Then there exists an $N_{1} \in \mathbb{N}$ such that for all $n>N_{1}, \sum_{i=k(n)}^{\infty} \mu_{i} / f_{r}(k(n))>$ $a / 2$. Therefore if we define $N_{2}(\delta)$ as $N_{2}(\delta)=\max \left(N_{1}, \min \left\{n \in N \mid k(n) \geq N_{0}\right\}\right)$, then for all $n>N_{2}(\delta), \sum_{i=k(n)}^{\infty} \lambda_{i} / f_{r}(k(n))<\delta$ and $f_{r}(k(n)) / \sum_{i=k(n)}^{\infty} \mu_{i}<2 / a$. That is, $\sum_{i=k(n)}^{\infty} \lambda_{i} / \sum_{i=k(n)}^{\infty} \mu_{i}<2 \delta / a$. This means $\underline{\lim }_{n \rightarrow \infty} \sum_{i=n}^{\infty} \lambda_{i} / \sum_{i=n}^{\infty} \mu_{i}=0$, which means $|\Psi\rangle$ cannot be convertible to $|\Phi\rangle$ by SLOCC from Vidal's Theorem.

Proof of part 2:

If $R^{+}(|\Phi\rangle)<R^{-}(|\Psi\rangle)$, then for all $R^{+}(|\Phi\rangle)<r<R^{-}(|\Psi\rangle), a \stackrel{\text { def }}{=} \underline{\lim }_{n \rightarrow \infty} \sum_{i=n}^{\infty} \lambda_{i} / f_{r}(n)>0$ and $\lim _{n \rightarrow \infty} \sum_{i=n}^{\infty} \mu_{i} / f_{r}(n)=0$. Then, for all $\delta>0$ there exists an $N_{0}(\delta)$ such that if $n>N_{0}(\delta), \sum_{i=n}^{\infty} \lambda_{i} / \sum_{i=n}^{\infty} \mu_{i}>a / 2 \delta$. That is $\lim _{n \rightarrow \infty} \sum_{i=n}^{\infty} \lambda_{i} / \sum_{i=n}^{\infty} \mu_{i}=\infty$. From Vidal's theorem, this means that $|\Psi\rangle$ can be converted to $|\Phi\rangle$ by SLOCC.

Hence, this SLOCC monotone satisfies the sufficient condition of convertibility of SLOCC at least with the above meaning. As we shall see in the following sections by using $R_{f_{r}}^{+}(|\Psi\rangle)$ and $R_{f_{r}}^{-}(|\Psi\rangle)$ together, we can determine the classification of SLOCC convertibility better than in the case of using the other SLOCC monotones, although we also need both $R_{f_{r}}^{+}$and $R_{f_{r}}^{-}$to lead to the sufficient condition. Therefore, we can consider this pair of monotones as extensions of the Schmidt rank.

In the last part of this subsection we note one important fact which we can easily see from Theorem 3, that is that "in infinite dimensional systems there are at least continuously infinitely many different classes of SLOCC convertibility." Since $R^{+}(|\Psi\rangle)\left(\right.$ or $\left.R^{-}(|\Psi\rangle)\right)$ is an SLOCC monotone whose range is a non-trivially connected set (interval) of real numbers, if $\left|\Psi_{r}\right\rangle$ satisfies $R^{+}\left(\left|\Psi_{r}\right\rangle\right)=r$, each $\left|\Psi_{r}\right\rangle$ should belong to different classes of SLOCC convertibility for every different value of $r$. That is, there exists an injective map from a non-trivially connected set of real numbers to the quotient set of states by SLOCC. Therefore, in infinitedimensional bipartite systems, the cardinal number of the quotient set of states by SLOCC convertibility is greater than or equal to the cardinal number of the continuum, (where the cardinal number of the continuum is equal to the cardinal number of an arbitrary interval of 
real numbers) 17. Comparing this to the finite-dimensional systems case, where the cardinal number of the quotient set of states by SLOCC convertibility is equal to the dimension of the local systems. This fact is remarkable, that is, the cardinal number of such classes is actually larger than the local dimension (which is only countably infinite) in infinite-dimensional systems.

\subsection{Examples of $R^{+}(|\Psi\rangle)$ and $R^{-}(|\Psi\rangle)$}

In this subsection we construct important examples of the SLOCC monotones $R^{+}(|\Psi\rangle)$ and $R^{-}(|\Psi\rangle)$, and analyze SLOCC convertibility between some interesting classes of genuinely infinite-dimensional states. One is a class of states with polynomially-damped Schmidt coefficients; another is the class of two-mode squeezed states. Since these new monotones depend on a real parameterized family of sequences $\left\{f_{r}(n)\right\}_{r \in(a, b)}$, we need to choose this family suitably to analyze SLOCC convertibility among particular states. For this purpose it is convenient to derive the reference-states class $\left\{\left|\Psi_{r}\right\rangle\right\}_{r \in(a, b)}$ for particular $\left\{f_{r}(n)\right\}_{r \in(a, b)}$, as the states which satisfy the condition $R^{+}\left(\left|\Psi_{r}\right\rangle\right)=R^{-}\left(\left|\Psi_{r}\right\rangle\right)=r$. Therefore, at first we construct a way of finding the reference class $\left|\Psi_{r}\right\rangle$ from $f_{r}(n)$. The following corollary gives a method.

Corollary 1 If $\left\{f_{r}(n)\right\}_{r \in(a, b), n \in \mathbb{N}}$ satisfies following conditions:

1. $\forall r \in(a, b), n_{1} \leq n_{2} \Rightarrow f_{r}\left(n_{1}\right)>f_{r}\left(n_{2}\right)$ (monotonically decreasing)

2. $\forall r \in(a, b)$ and $\forall n \in \mathbb{N}, f_{r}(n)+f_{r}(n+2) \geq 2 f_{r}(n+1)$ (convexity)

3. $\forall m \in \mathbb{N}, r_{1} \leq r_{2} \Leftrightarrow \lim _{n \rightarrow \infty} \frac{f_{r_{1}}(n)}{f_{r_{2}}(n+m)}=0$ (monotonicity),

then, $\left|\Psi_{r}\right\rangle=\frac{1}{c_{r}} \sum_{n=1}^{\infty} \sqrt{-f_{r}^{\prime}(n)}|n\rangle \otimes|n\rangle$, where $c_{r}=\sum_{n=1}^{\infty}-f_{r}^{\prime}(n)$, satisfies $R^{+}\left(\left|\Psi_{r}\right\rangle\right)=$ $R^{-}\left(\left|\Psi_{r}\right\rangle\right)=r$ which are made from $\left\{f_{r}(n)\right\}_{r \in \mathbb{R}, n \in \mathbb{N}}$, and where $f_{r}^{\prime}(x)$ denotes the derivative of $f_{r}(x)$ with respect to $x$.

Proof From conditions 1 and 2 above, there exists a class of doubly differentiable functions $\left\{f_{r}(x)\right\}_{r \in(a, b), x \in \mathbb{R}^{+}}$which are an extension of the sequences $\left\{f_{r}(n)\right\}_{r \in(a, b), n \in \mathbb{N}}$ such that they satisfy $f_{r}^{\prime}(x)<0$ and $f_{r}^{\prime \prime}(x) \geq 0$. Therefore, a class of states $\left\{\left|\Psi_{r}\right\rangle\right\}_{r \in(a, b)}$ is well defined and their Schmidt coefficients are $\left\{-f_{r}^{\prime}(n) / c_{r}\right\}_{n=1}^{\infty}$ in decreasing order. By definition then

$$
\begin{aligned}
\int_{n}^{\infty} \frac{-f_{r}^{\prime}(x)}{c_{r}} d x & \leq \sum_{k=n}^{\infty} \frac{-f_{r}^{\prime}(n)}{c_{r}} \leq \int_{n-1}^{\infty} \frac{-f_{r}^{\prime}(x)}{c_{r}} d x \\
\frac{f_{r}(n)}{c_{r} f_{r_{1}}(n)} & \leq \sum_{k=n}^{\infty} \frac{-f_{r}^{\prime}(n)}{c_{r} f_{r_{1}}(n)} \leq \frac{f_{r}(n-1)}{c_{r} f_{r_{1}}(n)} .
\end{aligned}
$$

If $r<r_{1}$ then

$$
\lim _{n \rightarrow \infty} \sum_{k=n}^{\infty} \frac{-f_{r}^{\prime}(n)}{c_{r} f_{r_{1}}(n)} \leq \lim _{n \rightarrow \infty} \frac{f_{r}(n-1)}{c_{r} f_{r_{1}}(n)}=0,
$$

and if $r>r_{2}$ then

$$
\lim _{n \rightarrow \infty} \sum_{k=n}^{\infty} \frac{-f_{r}^{\prime}(n)}{c_{r} f_{r_{1}}(n)} \geq \lim _{n \rightarrow \infty} \frac{f_{r}(n)}{c_{r} f_{r_{1}}(n)}=+\infty .
$$

Thus, $R^{+}\left(\left|\Psi_{r}\right\rangle\right)=R^{-}\left(\left|\Psi_{r}\right\rangle\right)=r$. 
This Corollary means that with the above three additional conditions for $f_{r}(n)$ we may always derive a class of reference states which correspond to each value of $R^{-}(|\Psi\rangle)$ and $R^{+}(|\Psi\rangle)$.

In what follows we construct examples of SLOCC monotones by means of the above Corollary and analyze two remarkable classes of states. One corresponds to the states which belong a higher class of SLOCC convertibility and the other to the well-known two-mode squeezed states.

As a first example consider $R^{-}(|\Psi\rangle)$ and $R^{+}(|\Psi\rangle)$ made from $\left\{f_{r}(n)=n^{-\left(\frac{1}{r}-1\right)}\right\}_{r \in(0,1)}$. By Corollary 1, their class is

$$
\left|\Psi_{r}\right\rangle=\frac{1}{\sqrt{\zeta(1 / r)}} \sum_{n=1}^{\infty} n^{-\frac{1}{2 r}}|n\rangle \otimes|n\rangle,
$$

where $\zeta(x)$ is the Riemann zeta function as a normalization factor. By definition, $R^{-}(|\Psi\rangle)$ and $R^{+}(|\Psi\rangle)$ represent how quickly the Schmidt coefficients of $|\Psi\rangle$ converge to 0 as a polynomiallydamped function. Thus, this function has a strictly positive value for states with polynomiallydamped Schmidt coefficients. Similarly, for all states $|\Psi\rangle$ for which the Schmidt coefficients damp exponentially like two-mode squeezed states, we have $R^{-}(|\Psi\rangle)=R^{+}(|\Psi\rangle)=0$. Because the Schmidt coefficients can never be proportional to $1 / n$ asymptotically in infinitedimensional systems (since $\sum_{n=1}^{\infty} 1 / n=\infty$ ), for small $\epsilon>0,\left|\Psi_{r}\right\rangle$ with $r=1-\epsilon$ can be converted to almost any state. In the above sense we can say that they belong to a "higher rank" of entanglement class in terms of single-copy SLOCC. On the other hand, the above state with small $\epsilon$ is not of the "highest states". That is, we can consider a class of states which belong to a higher rank of entanglement class than $\left\{\left|\Psi_{r}\right\rangle\right\}_{r \in(0,1)}$ as follows. For the states

$$
\left|\Psi_{t}\right\rangle=\frac{1}{C_{t}} \sum_{n=1}^{\infty} \frac{1}{\sqrt{x(\log x)^{t}}}|n\rangle \otimes|n\rangle
$$

with $t>0, R^{-}\left(\left|\Psi_{t}\right\rangle\right)=R^{+}\left(\left|\Psi_{t}\right\rangle\right)=1$, and we can easily see that for all $t>0,\left|\Psi_{r}\right\rangle$ can not be converted to $\left|\Psi_{t}\right\rangle$ by SLOCC. In a similar manner, for all one-parameter classes of states we can always define a class of states which belong to a higher rank and can define a new pair of monotones from this class of states. Therefore, there does not exist a highest one-parameter class of states within the SLOCC classification.

As a next example consider $f_{q}(n)=e^{2 n \log q}=q^{2 n}, q \in(0,1)$. In this case, the reference class is $\left|\Psi_{q}\right\rangle=\frac{1}{c_{q}} \sum_{n=1}^{\infty} q^{n}|n\rangle \otimes|n\rangle$, that is the well-known two-mode squeezed states with $\frac{1}{2} \log \frac{1+q}{1-q}$ being the squeezing parameter. Therefore, $R_{f_{q}}^{+}(|\Psi\rangle)$ and $R_{f_{q}}^{-}(|\Psi\rangle)$ can be regarded as being analogs of squeezing parameters for any entangled states.

The above two examples also show that the classification of SLOCC is quite different from the classification by the amount of entanglement, that is, the classification of asymptotic (infinite-copy) LOCC in infinite-dimensional systems. In infinite-dimensional systems we often consider the class of two-mode squeezed states $\left|\Psi_{q}\right\rangle=\frac{1}{c_{q}} \sum_{n=1}^{\infty} q^{n}|n\rangle \otimes|n\rangle$ with $q=1-\epsilon$ instead of the maximally entangled states. Because $\lim _{q \rightarrow 1} E\left(\left|\Psi_{q}\right\rangle\right)=\infty$ this state converts to almost any state asymptotically by infinite-copy LOCC with unit probability. However by single-copy SLOCC with non-zero probability they cannot be converted to states $|\Psi\rangle$ with $R^{+}(|\Psi\rangle)>0$, where the monotone $R^{+}(|\Psi\rangle)$ is made from $f_{r}(n)=n^{-\left(\frac{1}{r}-1\right)}$. On the other hand, if we consider the class of states $\left|\Psi_{r}\right\rangle=\sum_{n=1}^{\infty} n^{-\frac{1}{2 r}}|n\rangle \otimes|n\rangle / \sqrt{\zeta(1 / r)}$ as we have 
already seen for small $\epsilon>0,\left|\Psi_{1-\epsilon}\right\rangle$ can be converted to almost any state by single-copy SLOCC with non-zero probability. Although the amount of entanglement for both $\left\{\left|\Psi_{q}\right\rangle\right\}$ and $\left\{\left|\Psi_{r}\right\rangle\right\}$ tend to infinity in the limit, $\left\{\left|\Psi_{r}\right\rangle\right\}$ belongs to a higher class than $\left\{\left|\Psi_{q}\right\rangle\right\}$ in the single-copy scenario.

We add one final remark here: Although, we have only presented two examples for $f_{r}(n)$, there may be many other examples which are important in some situations. Generally speaking, for any given states, we can find a suitable function $f_{r}(n)$ for the analysis of the states. For example, if we deal with states whose Schmidt coefficients damp exponentially we can chose $f_{r}(n)=\exp \left(n^{-1 / r}\right), \exp \left(\exp \left(n^{-1 / r}\right)\right)$, etc, as the coefficients damp quickly enough to evaluate the monotones for the states.

\subsection{Strong inhibition law}

So far we have emphasized the difference between states with exponentially-damped Schmidt coefficients and those with polynomially-damped coefficients and shown that exponentiallydamped states cannot be converted into polynomially-damped states no matter how large their measure of entanglement is. Here we shall give one more fact which will demonstrate the remarkable difference between exponentially and polynomially-damped states. That is, "However finitely many copies there are, exponentially-damped states cannot be converted into polynomially-damped states." This fact can be showed as the follows: Suppose $|\Psi\rangle$ is an exponentially-damped state and $|\Phi\rangle$ is a polynomially-damped one, then rigorously speaking, there exists a real number $r$ and a polynomial $p(n)$ which satisfy $\lim _{n \rightarrow \infty} \frac{g_{|\Psi\rangle}(n)}{e^{-r n}}=0$ and $\varliminf_{n \rightarrow \infty} \frac{p(n)}{g_{|\Phi\rangle}(n)}=0$, where $g_{|\Psi\rangle}(n)$ is Vidal's monotone of $|\Psi\rangle$. Define $\left|\xi_{r}\right\rangle=\frac{1}{C_{r}} \sum_{n=1}^{\infty} e^{-r n}|n\rangle \otimes$ $|n\rangle$, then we have

$$
\left|\xi_{r}\right\rangle^{\otimes p}=\frac{1}{C_{r}^{p}} \sum_{n_{1}, n_{2}, \cdots, n_{p}}^{\infty} e^{-r\left(n_{1}+n_{2}+\cdots+n_{p}\right)}\left|n_{1}, n_{2}, \cdots, n_{p}\right\rangle \otimes\left|n_{1}, n_{2}, \cdots, n_{p}\right\rangle .
$$

If we reorder the Schmidt terms to the form $\left|\xi_{r}\right\rangle^{\otimes p}=\frac{1}{C} \sum_{k=1}^{\infty} f(k)|k\rangle \otimes|k\rangle$ we can see by easy calculation that $f(k) \leq e^{-r\left[(p ! k)^{1 / p}+1\right]}$. Thus, we have $\lim _{n \rightarrow \infty} f(n) / p(n)=0$ and this means $|\Psi\rangle^{\otimes p}$ cannot converted into $|\Phi\rangle$ for any $p \in \mathbb{N}$. This result shows that in infinite-dimensional systems some classes of states (like states with finite Schmidt ranks, with exponentiallydamped Schmidt coefficients, and with polynomially-damped Schmidt coefficients) can be distinguished from each other more strongly than the case of finite-dimensional systems by SLOCC classification. Thus, with arbitrary finitely-many copies, we also cannot convert the states from finite rank to infinite rank, and similarly from exponentially damped to polynomially damped. In finite-dimensional systems, there is no feature like this. Therefore, these properties of entanglement are genuine for infinite-dimensional systems and show the special strong position of states with polynomially-damped Schmidt coefficients from the view of finite-copy transformations.

As a final remark for this section we must discuss the energy of such long-tailed states. In realistic situations the set of states which can be produced experimentally will be limited by some bound in energy. Therefore, it is essential to consider the subset of states which consist of states restricted to that bounded energy. However, for several states with polynomiallydamped Schmidt coefficients, the mean value of a polynomial Hamiltonian, like for example 
the harmonic oscillator, diverges. Therefore, generally only a fraction of polynomially-damped states can be created in laboratories.

\section{Summary}

In this paper in order to avoid the difficulties of discontinuity and infinite amounts of classical communication in the theory of SLOCC convertibility of infinite-dimensional systems, we proposed a new definition of convertibility, $\epsilon$-convertibility, as the convertibility of states in an approximated setting by means of the trace norm. In the Section 2 we showed that this definition guarantees at least weak continuity for SLOCC convertibility (Lemma 1), and guarantees that the protocol only uses finite amounts of classical communication. Then, we reconstructed the basic theorems of single-copy LOCC and SLOCC transformation, Neilsen's and Vidal's theorem in the infinite-dimensional pure state space (Theorems 1 and 21). As a result we showed that under this change of definition the framework of entanglement convertibility is preserved for infinite-dimensional systems, and therefore, our definition of $\epsilon$-convertibility for LOCC is suitable and sufficient for realistic conditions of quantum information processing in infinite-dimensional systems.

In Section 3 in order to study SLOCC convertibility in infinite-dimensional systems, we constructed a pair of SLOCC monotones which can be considered as extensions of the Schmidt rank to infinite-dimensional spaces. By these monotones we showed that states with polynomially-damped Schmidt coefficients belong to a higher rank of entanglement class than other states in terms of single-copy SLOCC convertibility.

In the last Section 3.3 we showed that arbitrary finitely many copies of exponentiallydamped states cannot be converted to even a single copy of polynomially-damped states. Since such differences of classes do not exist in the finite-dimensional setting, the SLOCC classification of infinite-dimensional states has a much richer structure than for finite-dimensional ones. Therefore, these new features of entanglement have the potential to produce new quantum information protocols which are impossible for finite-dimensional systems. Finally, we stress that in infinite-dimensional systems, there remain important problems that are yet to be solved even for the simplest bipartite pure states.

\section{Acknowledgements}

MO is grateful to Professor M. Ozawa, Professor M.B. Plenio, Professor K. Matsumoto, Professor M. Hayashi, and Dr. A. Miyake for discussions. This work has been supported by the Asashi Grass Foundation, the Sumitomo Foundation, the Japan Society of Promotion of Science, the Japan Scholarship Foundation, the Japan Science and Technology Agency, and the Special Coordination Funds for Promoting Science and Technology.

\section{References}

1. A. Einstein, B. Podolsky, and N. Rosen Phys. Rev. 47, 777 (1935); J.S. Bell Physics 1, 195 (1964); J.F. Clauser, M.A. Horne, A. Shimony. R.A. Holt Phys. Rev. Lett. 23, 880 (1969); R.F. Werner, Phys. Rev. A 404277 (1989).

2. A.K. Ekert, Phys. Rev. Lett. 68, 661 (1991); P.W. Shor, Proc. 35nd Annual Symposium on Foundations of Computer Science, (IEEE Computer Society Press, 1994), 124-134.

3. C.H. Bennett and S.J. Wiesner Phys. Rev. Lett. 69, 2884 (1992); C.H. Bennett, G. Brassard, C. Crepeau, R. Jozsa, A. Peres, and W.K. Wootters, Phys. Rev. Lett. 70, 1895 (1993). 
4. J. von Neumann, Mathematical Foundations of Quantum Mechanics (Princeton University Press, Princeton, New Jersey, 1955).

5. M. Reed, B. Simon Functional Analysis (Methods of Modern Mathematical Physics) (Academic Press, 1980).

6. C.H. Bennett, D.P. DiVincenzo, J.A. Smolin and W.K. Wootters, Phys. Rev. A 54, 3824 (1996); C.H. Bennett, G. Brassard, S. Popescu, B. Schumacher, J.A. Smolin and W.K. Wootters, Phys. Rev. Lett. 76, 722 (1996); C.H. Bennett, H.J. Berstein, S. Popescu and B. Schumacher, Phys. Rev. A 53, 2046 (1996).

7. M.A. Nielsen, Phys. Rev. Lett. 83, 436 (1999).

8. G. Vidal, Phys. Rev. Lett. 83, 1046 (1999).

9. G. Vidal J. Mod. Opt. 47, 355 (2000).

10. L.-M. Duan, G. Giedke, J.I. Cirac, and P. Zoller, Phys. Rev. Lett. 84, 2722 (2000); R. Simon, Phys. Rev. Lett. 84, 2726 (2000); G. Giedke, B. Kraus, M. Lewenstein, J.I. Cirac, Phys. Rev. A 64, 052303 (2001).

11. G. Giedke, J. Eisert, J.I. Cirac and M.B. Plenio, Quant. Inf. Comp. 3, 211 (2003); G. Giedke, M.M. Wolf, O. Kruger, R.F. Werner and J.I. Cirac, Phys. Rev. Lett. 91, 107901 (2003); M.M. Wolf, G. Giedke, O. Kruger, R.F. Werner and J.I. Cirac, quant-ph/0306177 (2003).

12. G. Giedke and J.I. Cirac Phys. Rev. A 66, 032316 (2002); J. Fiurasek Phys. Rev. Lett. 89, 137904 (2002); J. Eisert, S. Scheel and M.B. Plenio, Phys. Rev. Lett. 89, 137903 (2002); J. Eisert, D. Browne, S. Scheel and M.B. Plenio, Ann. Phys. (NY) 311, 431 (2004).

13. J. Eisert, C. Simon, and M.B. Plenio, J. Phys. A 35, 3911, (2002); M. Keyl, D. Schlingemann, and R.F. Werner, Quant. Inf. Comp. 3, 281 (2003); M.M. Wolf, G. Giedke, O. Krueger, R.F. Werner, J.I. Cirac Phys. Rev. A 69, 052320 (2004).

14. V. Vedral, M.B. Plenio, M.A. Rippin, P.L. Knight, Phys. Rev. Lett. 78, 2275 (1997); E.M. Rains, IEEE Trans. Inf. Tec. 47, 2921 (2001).

15. M.J. Donald, M. Horodecki, O. Rudolph, J. Math. Phys. 43, 4252 (2002).

16. H.-K. Lo and S. Popescu, Phys. Rev. A 63, 022301 (2001).

17. A.N. Kolmogorov and S.V. Fomin, Introductory real analysis (Dover Publications, Inc, 1975)

18. R. Bhatia, Matrix analysis (Springer-Verlag, New York, 1997).

19. G.M. D'Ariano, M.F. Sacchi, Phys. Rev. A 67, 042312 (2003).

20. A.S. Markus, Russian Math. Surveys 19, 91 (1964)

\section{Appendix A Schmidt decomposition and Lo-Popescu's Theorem in infinite di- mensional systems}

In this appendix and the next, as a preparation for the proofs of Nielsen's and Vidal's theorem in infinite dimensional systems, we will see how we can extend basic theorems about LOCC and majorization [7, 8 to infinite dimensional systems.

At first, we extend the concept of Schmidt decomposition and Schmidt coefficients in infinite-dimensional systems:

Theorem 4 (Schmidt decomposition) For any $|\Psi\rangle \in \mathcal{H}=\mathcal{H}_{A} \otimes \mathcal{H}_{B}$, there exist orthonormal sets (but not necessarily basis sets) $\left\{\left|e_{i}\right\rangle\right\}_{i=1}^{\infty}$ and $\left\{\left|f_{i}\right\rangle\right\}_{i=1}^{\infty}$ of $\mathcal{H}_{A}$, and $\mathcal{H}_{B}$, respectively, such that

$$
|\Psi\rangle=\sum_{i=1}^{\infty} \sqrt{\lambda_{i}}\left|e_{i}\right\rangle \otimes\left|f_{i}\right\rangle,
$$

where $\lambda_{i} \geq 0, \lambda_{i} \geq \lambda_{i+1}$ and $\sum_{i=1}^{\infty} \lambda_{i}=1$. The representation of a state $|\Psi\rangle$ in the form of Eq.(A.1) is called a Schmidt decomposition and $\left\{\lambda_{i}\right\}_{i=1}^{\infty}$ are called Schmidt coefficients in infinite-dimensional systems. 
Proof We use the singular value decomposition given as follows in an infinite dimensional system: For a compact operator $M$ from $\mathcal{H}_{A}$ onto $\mathcal{H}_{B}$, there exist orthonormal sets (but not necessarily basis sets) $\left\{\left|e_{i}\right\rangle\right\}_{i=1}^{\infty} \subset \mathcal{H}_{A}$ and $\left\{\left|f_{i}\right\rangle\right\}_{i=1}^{\infty} \subset \mathcal{H}_{B}$ and positive real numbers $\left\{\lambda_{i}\right\}_{i=1}^{\infty}$ with $\sqrt{\lambda_{n}} \rightarrow 0$ such that

$$
M=\sum_{i=1}^{\infty} \sqrt{\lambda_{i}}\left|e_{i}\right\rangle\left\langle f_{i}\right|,
$$

where the above sum converges in the operator norm [5]. In particular, if $M$ is a HilbertSchmidt class operator, $\left\{\sqrt{\lambda_{i}}\right\}_{i=1}^{\infty}$ satisfy $\sum_{i=1}^{\infty} \lambda_{i}=\left(\|M\|_{2}\right)^{2} \stackrel{\text { def }}{=} \operatorname{tr} M^{\dagger} M$, where $\|\cdot\|_{2}$ is the Hilbert-Schmidt norm [5. Thus, we derive Eq. (A.1) from Eq. (A.2), because the linear map $\left|e_{i}\right\rangle\left\langle f_{j}|\mapsto| e_{i}\right\rangle \otimes\left|f_{j}\right\rangle$ gives an isomorphism from the Hilbert-Schmidt space $\mathfrak{C}_{2}\left(\mathcal{H}_{A}, \mathcal{H}_{B}\right)$ (the Hilbert space of all Hilbert-Schmidt class operators between $\mathcal{H}_{A}$ and $\mathcal{H}_{B}$ with the inner product $\left.(M \mid N) \stackrel{\text { def }}{=} \operatorname{tr} M^{\dagger} N\right)$ to the Hilbert space $\mathcal{H}=\mathcal{H}_{A} \otimes \mathcal{H}_{B}[5]$.

In finite $d$-dimensional bipartite systems, the Schmidt decomposition of a state $|\psi\rangle$ is given by

$$
|\psi\rangle=\sum_{i=1}^{d} \sqrt{\lambda_{i}}\left|e_{i}\right\rangle \otimes\left|f_{i}\right\rangle,
$$

where $\left\{\left|e_{i}\right\rangle\right\}_{i=1}^{d}$ and $\left\{\left|f_{i}\right\rangle\right\}_{i=1}^{d}$ are the basis sets. Therefore, convertibility of states under local unitary operations are determined by the Schmidt coefficients $\left\{\lambda_{i}\right\}_{i=1}^{d}$ of states, namely, the two states are convertible to each other under local unitary operations if and only if the two states have same Schmidt coefficients. In infinite-dimensional systems, the Schmidt coefficients determine convertibility of states under local partial isometry instead of local unitary operations. That is, if the Schmidt coefficients of $|\Psi\rangle$ and $|\Phi\rangle$ are the same, then there exist local partial isometries $U_{A}$ and $U_{B}$ and $|\Psi\rangle=U_{A} \otimes U_{B}|\Phi\rangle$ is satisfied.

Partial isometry is defined as a unitary operator between subspaces. If we had defined the Schmidt coefficients to be a sequence including the dimension of the kernel of the reduced density matrix (of the given state), we could make Schmidt coefficients indicating the convertibility under local unitary operations. However, to develop the theory of LOCC and SLOCC, (which include local partial isometries), convertibility for infinite-dimensional systems, the former definition is more suitable than the latter, so we take the definition of Eq. A.1 This is because states are convertible to each other by LOCC, if and only if they are convertible to each other by local partial isometries (we can show this fact from Theorem 2); moreover, there exists a pair of states which are convertible to each other by local partial isometries, but not by local unitaries. The following example satisfies such a condition. Suppose states $|\Psi\rangle$ and $|\Phi\rangle$ on $\mathcal{H}_{A} \otimes \mathcal{H}_{B}$ are defined as

$$
\begin{aligned}
& |\Psi\rangle=\sum_{i=1}^{\infty} \sqrt{\lambda_{i}}\left|e_{i}\right\rangle \otimes\left|f_{i}\right\rangle \\
& |\Phi\rangle=\sum_{i=1}^{\infty} \sqrt{\lambda_{i}}\left|e_{2 i}\right\rangle \otimes\left|f_{2 i}\right\rangle,
\end{aligned}
$$

where $\left\{\left|e_{i}\right\rangle\right\}_{i=1}^{\infty}$ and $\left\{\left|f_{i}\right\rangle\right\}^{\infty}$ are orthonormal basis sets of $\mathcal{H}_{A}$ and $\mathcal{H}_{B}$, respectively. In this case, $|\Phi\rangle$ and $|\Psi\rangle$ can be convertible to each other by LOCC: $|\Phi\rangle$ can be convertible to $|\Psi\rangle$ by 
a local partial isometry $\sum_{i=1}^{\infty}\left|e_{i}\right\rangle\left\langle e_{2 i}|\otimes| f_{i}\right\rangle\left\langle f_{2 i}\right|$, and $|\Psi\rangle$ can be convertible to $|\Phi\rangle$ by a local isometry $\sum_{i=1}^{\infty}\left|e_{2 i}\right\rangle\left\langle e_{i}|\otimes| f_{2 i}\right\rangle\left\langle f_{i}\right|$. However, their are not convertible by local unitaries. This is because a subspace spanned by $\left\{\left|e_{2 i}\right\rangle\right\}_{i=1}^{\infty}$ and a subspace spanned by $\left\{\left|f_{2 i}\right\rangle_{i=1}^{\infty}\right.$ should be mapped to $\mathcal{H}_{A}$ and $\mathcal{H}_{B}$, respectively; a proper subspace should be mapped to a whole space. This map is obviously impossible by unitary operators, which are bijections and always map a whole space to a whole space.

Actually, from a physical point of view, convertibility under local partial isometry can be understood that we may need additional independent ancilla systems for each subspace to convert $|\Psi\rangle$ to $|\Phi\rangle$. For example, $|\Phi\rangle$ defined as Eq. A.5. can be transformed to $|\Psi\rangle$ defined as Eq. A.4 by the following protocol: First, attach one-qubit ancilla systems $\mathcal{H}_{A^{\prime}}$ and $\mathcal{H}_{B^{\prime}}$ to both local systems $\mathcal{H}_{A}$ and $\mathcal{H}_{B}$, and prepare the ancilla systems in $|0\rangle_{A^{\prime}}$ and $|0\rangle_{B^{\prime}}$. Second, apply a local unitary transformation $U_{A A^{\prime}} \otimes U_{B B^{\prime}}$ to the states $|\Phi\rangle_{A B} \otimes|0\rangle_{A^{\prime}} \otimes|0\rangle_{B^{\prime}}$, where $U_{A A^{\prime}}$ and $U_{B B^{\prime}}$ are unitary transformations on $\mathcal{H}_{A} \otimes \mathcal{H}_{A^{\prime}}$ and $\mathcal{H}_{B} \otimes \mathcal{H}_{B^{\prime}}$ defined as

$$
\begin{gathered}
U_{A A^{\prime}} \stackrel{\text { def }}{=} \sum_{n=1}^{\infty}\left(| n \rangle _ { A } | 0 \rangle _ { A ^ { \prime } A } \langle 2 n | _ { A ^ { \prime } } \langle 0 | + | 2 n + 1 \rangle _ { A } | 1 \rangle _ { A ^ { \prime } A } \left\langle2 n+\left.1\right|_{A^{\prime}}\langle 0|\right.\right. \\
+|2 n\rangle_{A}|1\rangle_{A^{\prime} A}\left\langle\left. n\right|_{A^{\prime}}\langle 1|\right) \\
U_{B B^{\prime}} \stackrel{\text { def }}{=} \sum_{n=1}^{\infty} \begin{array}{c}
\left(| n \rangle _ { B } | 0 \rangle _ { B ^ { \prime } B } \langle 2 n | _ { B ^ { \prime } } \langle 0 | + | 2 n + 1 \rangle _ { B } | 1 \rangle _ { B ^ { \prime } B } \left\langle2 n+\left.1\right|_{B^{\prime}}\langle 0|\right.\right. \\
+|2 n\rangle_{B}|1\rangle_{B^{\prime} B}\left\langle\left. n\right|_{B^{\prime}}\langle 1|\right) .
\end{array}
\end{gathered}
$$

After this local unitary transformation, the state is changed to $|\Psi\rangle_{A B} \otimes|0\rangle_{A^{\prime}} \otimes|0\rangle_{B^{\prime}}$. Finally, by removing the ancilla system $\mathcal{H}_{A^{\prime}}$ and $\mathcal{H}_{B^{\prime}}$, we derive $|\Psi\rangle$ on the systems $\mathcal{H}_{A} \otimes \mathcal{H}_{B}$.

For finite-dimensional systems, Lo and Popescu proved that if $|\Psi\rangle$ can be converted into $|\Phi\rangle$ by LOCC, there exists a one-way classical communication LOCC which consists of a local measurement of one of the local spaces and a unitary operation of the other depending on the result of the measurement [16. This theorem is called the Lo-Popescu theorem. Intuitively speaking, the Schmidt decomposition denotes the existence of symmetry between local subspaces, and Lo-Popescu's theorem is the reflection of the symmetry of subsystems. As we have shown that the Schmidt decomposition in infinite-dimensional systems is weaker (indicating equivalence under partial isometries instead of unitary operations) than finitedimensional systems, the corresponding Lo-Popescu theorem is slightly modified as follows.

Theorem 5 (Lo-Popescu's) In the separable Hilbert space $\mathcal{H}_{A} \otimes \mathcal{H}_{B}$, for any given state $|\Psi\rangle$ and bounded operator $M \in \mathfrak{B}\left(\mathcal{H}_{B}\right)$ there exist a bounded operator $N \in \mathfrak{B}\left(\mathcal{H}_{A}\right)$ and partial isometry $U \in \mathfrak{B}\left(\mathcal{H}_{B}\right)$ which satisfy $I \otimes M|\Psi\rangle=N \otimes U|\Psi\rangle$.

Proof Suppose $|\Psi\rangle=\sum_{i=1}^{\infty} \sqrt{\mu_{i}}\left|a_{i}\right\rangle \otimes\left|b_{i}\right\rangle$. Define a partial isometry $U$ as $U=\sum_{i=1}^{\infty}\left|b_{i}\right\rangle\left\langle a_{i}\right|$, then we have

$$
I \otimes M|\Psi\rangle=\sum_{i=1}^{\infty} \sqrt{\mu_{i}}\left|a_{i}\right\rangle \otimes M\left|b_{i}\right\rangle
$$

and

$$
M U \otimes I|\Psi\rangle=\sum_{i=1}^{\infty} \sqrt{\mu_{i}} M\left|b_{i}\right\rangle \otimes\left|b_{i}\right\rangle
$$


Thus, we obtain

$$
\operatorname{tr}_{A} I \otimes M|\Psi\rangle\left\langle\Psi\left|I \otimes M^{\dagger}=\operatorname{tr}_{B} M U \otimes I\right| \Psi\right\rangle\langle\Psi| U^{\dagger} M^{\dagger} \otimes I .
$$

By our definition of Schmidt decomposition, $\rho_{A}$ and $\rho_{B}$ are partial isometry equivalent for any state $|\Psi\rangle$. Therefore, there exist partial isometries $U_{A}$ and $U_{B}$ which satisfy

$$
U_{A} \otimes U_{B}(M U \otimes I)|\Psi\rangle=I \otimes M|\Psi\rangle .
$$

Defining $N \stackrel{\text { def }}{=} U_{A} M U \otimes U_{B}$, then the theorem has been proven.

\section{Appendix B Majorization in infinite-dimensional systems}

In finite-dimensional systems, majorization is a pseudo partial ordering on the whole vector space [18. On the other hand in infinite-dimensional system, majorization can be defined on only a subset of the whole vector space. To formulate our definition of Schmidt coefficients in infinite-dimensional systems, we define a majorization on

$$
l_{1}=\left\{\left\{x_{i}\right\}_{i=1}^{\infty}\left|\sum_{i=1}^{\infty}\right| x_{i} \mid<\infty\right\} .
$$

For mathematical simplicity, we only define majorization on

$$
l_{1}^{+}=\left\{\{x\}_{i=1}^{\infty} \in l_{1} \mid x_{i} \geq 0, \sharp\left\{i \mid x_{i}=0\right\}<\infty \vee \sharp\left\{i \mid x_{i}>0\right\}<\infty\right\},
$$

where $\sharp$ denotes the cardinality of a set. In this case $l_{1}^{+}$is a convex cone of $l_{1}$ and identifies the set of all permutations of Schmidt coefficients. Thus, $l_{1}^{+}$is enough for our purposes, and we do not need to define majorization for all element of $l_{1}$. Moreover, in the following definition of majorization, we use decreasing reordering of $x \in l_{1}$. However, if $x$ is not in $l_{1}^{+}$, it is difficult to rearrange elements of $x$ in decreasing order, and we need to extend the definition of majorization so as to include sequences which are not in $l_{1}^{+}$, but in $l_{1} \cdot{ }^{a}$ This is also the reason why we define majorization only on $l_{1}^{+}$.

Now, we define majorization of Schmidt coefficients in infinite-dimensional systems as follows:

Definition 4 For any $x, y \in l_{1}^{+}, x \prec_{\omega} y$ (or $x$ is sub-majorized by $y$ ) is defined, if and only if

$$
\sum_{i=1}^{k} x_{i}^{\downarrow} \leq \sum_{i=1}^{k} y_{i}^{\downarrow},
$$

for $k \in \mathbb{N}$, where $x_{i}^{\downarrow}$ is given by $x_{i}^{\downarrow}=x_{P(i)}$, and $P$ is an element of an infinite symmetry group satisfying $x_{i}^{\downarrow} \geq x_{i+1}^{\downarrow}$ in decreasing reordering of $x$. Similarly, if $x$ and $y$ satisfy,

$$
\sum_{i=k}^{\infty} x_{i}^{\downarrow} \geq \sum_{i=k}^{\infty} x_{i}^{\downarrow},
$$

${ }^{a}$ The general definition of majorization of sequences can be found in 20]. The all propositions in this subsection can be extended to this general case by appropriate modification of proofs. 
then, we write $x \prec^{\omega} y$ and say $x$ is super-majorized by $y$.

Additional to the sub-majorization or super-majorization conditions, (both conditions lead to the same majorization condition) if $x$ and $y$ satisfy the normalization condition

$$
\sum_{i=1}^{\infty} x_{i}^{\downarrow}=\sum_{i=1}^{\infty} y_{i}^{\downarrow}
$$

then we write $x \prec y$ and say $x$ is majorized by $y$.

The sub-majorization condition does not require the normalization condition, but it is easily proven that $\mathbf{x} \prec_{\omega} \mathbf{y}$ is equivalent to

$$
\sum_{j=1}^{\infty}\left(\mathbf{x}_{\mathbf{j}}^{\downarrow}-t\right)^{+} \leq \sum_{j=1}^{\infty}\left(\mathbf{y}_{\mathbf{j}}^{\downarrow}-t\right)^{+},
$$

for all real $t$, where $z^{+}=\max (z, 0)$ is the positive part of any real number.

Uhlmann's theorem relates operations on quantum states and majorization conditions. This theorem is one of the essential items for proving Nielsen's theorem for LOCC convertibility. To prove Uhlmann's theorem in infinite-dimensional systems, we define a doubly stochastic matrix in infinite-dimensional systems. It is similar to the one for finite-dimensional systems. For all double sequences $\left\{d_{i j}\right\}_{i j=1}^{\infty}$ which satisfy $\sum_{i=1}^{\infty} d_{i j}=1$ for all $j$, and $\sum_{j=1}^{\infty} d_{i j}=1$ for all $i$, we can define a bounded linear operator $D \in \mathfrak{B}\left(l_{1}\right)$ by

$$
D \mathbf{x}=\left\{\sum_{j=1}^{\infty} d_{i j} \mathbf{x}_{\mathbf{j}}\right\}_{i=1}^{\infty},
$$

for all $\mathbf{x} \in l_{1}$. These operators are called doubly stochastic matrices on $l_{1}$. We can easily see that the operator norm of a doubly stochastic matrix is 1 .

The defined doubly stochastic matrices are related to majorization as follows: If $D$ is doubly stochastic, then, for all $\mathrm{x} \in S$, we have

$$
D \mathbf{x} \prec \mathbf{x},
$$

since

$$
\begin{aligned}
\sum_{i=1}^{\infty}\left[(D x)_{i}-t\right]^{+} & =\sum_{i=1}^{\infty}\left[\sum_{j=1}^{\infty} D_{i j}\left(x_{j}-t\right)\right]^{+} \\
& \leq \sum_{i=1}^{\infty} \sum_{j=1}^{\infty} D_{i j}\left(x_{j}-t\right)^{+} \\
& =\sum_{j=1}^{\infty}\left(x_{j}-t\right)^{+}
\end{aligned}
$$

for any real $t$, due to the convexity of ()$^{+}$. On the other hand, $\sum_{i=1}^{\infty}(D x)_{i}=\sum_{i=1}^{\infty} x_{i}$ is trivial. Thus, the necessary condition (majorization condition) for the doubly stochastic condition is proven. We note that Eq.(B.7) is also valid for weaker conditions of $D$ than the doubly stochastic matrix, for example, $D$ satisfying $\sum_{j=1}^{\infty} d_{i j} \leq 1$.

Now we are ready to extend Uhlmann's theorem for infinite-dimensional systems: 
Theorem 6 (Uhlmann) If the two density operators $\rho_{1}$ and $\rho_{2}$ on the infinite separable Hilbert space $\mathcal{H}$ satisfy the following relation,

$$
\begin{array}{r}
\rho_{1}=\sum_{j=1}^{\infty} p_{j} U_{j} \rho_{2} U_{j}^{\dagger}, \\
\sum_{j=1}^{\infty} p_{j}=1,
\end{array}
$$

where $U_{j}$ are partial isometries whose initial space includes closure of the range of $\rho_{2}, \operatorname{kerU}_{\mathbf{j}}{ }^{\perp} \supset$ $\overline{\operatorname{Ran} \rho_{2}}$, then the non-zero eigenvalue of $\rho_{1}$ is majorized by $\rho_{2}$.

Proof Suppose $\rho_{1}=\sum_{k=1}^{\infty} \mu_{k}\left|e_{k}\right\rangle\left\langle e_{k}\right|$ and $\rho_{2}=\sum_{i=1}^{\infty} \lambda_{i}|i\rangle\langle i|$. Define a partial isometry as $V=\sum_{i=1}^{\infty}|i\rangle\left\langle e_{i}\right|$, whose initial space is given by $\overline{\operatorname{Ran} \rho_{1}}$, and whose final space is given by $\overline{\operatorname{Ran} \rho_{2}}$. Then $U_{j} V$ is a partial isometry whose initial and final space are given by $\overline{\operatorname{Ran} \rho_{1}}$ and $U_{j}\left(\overline{\operatorname{Ran} \rho_{2}}\right)$, respectively. Actually, it is trivial that $U_{j} V$ is a zero operator on $\overline{\operatorname{Ran} \rho}{ }^{\perp}$. Now suppose $|\Phi\rangle \in \overline{\operatorname{Ran} \rho_{1}}$, then we obtain $\| U_{j} V|\Phi\rangle\|=\||\Phi\rangle \|$ from the condition $V|\Phi\rangle \in \overline{\operatorname{Ran} \rho_{2}}$.

Next, define Fourier's coefficients of $U_{j}|i\rangle$ as $u_{j i}^{h}$, that is, $U_{j}|i\rangle=U_{j} V\left|e_{i}\right\rangle=\sum_{h=1}^{\infty} u_{j i}^{h}\left|e_{h}\right\rangle$, and

$$
\sum_{h=1}^{\infty}\left|u_{j i}^{h}\right|^{2}=1
$$

Then, we can rewrite Eq.(B.9) as,

$$
\sum_{k=1}^{\infty} \mu_{k}\left|e_{k}\right\rangle\left\langle e_{k}\right|=\sum_{i j \in N^{2}} p_{j} \lambda_{i}\left(\sum_{h=1}^{\infty} u_{j i}^{h}\left|e_{h}\right\rangle\right)\left(\sum_{l=1}^{\infty} u^{* l}{ }_{j i}\left\langle e_{l}\right|\right) .
$$

Taking the inner product between $\left|e_{n}\right\rangle$,

$$
\mu_{n}=\sum_{i j \in \mathbb{N}^{2}} p_{j} \lambda_{i}\left|u_{j i}^{n}\right|^{2}=\sum_{i=1}^{\infty} \lambda_{i} \sum_{j=1}^{\infty} p_{j}\left|u_{j i}^{n}\right|^{2} .
$$

We define $D_{n i}$ as $D_{n i}=\sum_{j=1}^{\infty} p_{j}\left|u_{j i}^{n}\right|^{2}$. (B.11) guarantees that $\sum_{n=1}^{\infty} D_{n i}=1$. Since

$$
\begin{aligned}
\sum_{i=1}^{\infty}\left|u_{j i}^{n}\right|^{2} & =\sum_{i=1}^{\infty}\left|\left\langle e_{n}\left|U_{j} V\right| e_{i}\right\rangle\right|^{2} \leq \| U_{j} V\left|e_{n}\right\rangle \|^{2} \\
& \leq\left\|U_{j}\right\|_{\text {op }}^{2}\|V\|_{\text {op }}^{2} \|\left|e_{n}\right\rangle \|^{2}=1,
\end{aligned}
$$

where $\|\cdot\|_{\text {op }}$ is the operator norm and $\sum_{i=1}^{\infty} D_{n i} \leq 1$. Therefore, using the necessary condition of the double stochastic matrices and the weaker condition of (B.7) for $D$, we derive $\mu=D \lambda \prec$ $\lambda$. The theorem is proven. 


\section{Appendix C Proof of Theorem 1 (Nielsen's theorem in infinite-dimensional systems)}

In this appendix, based on Appendix A and B, we prove Nielsen's [7] theorem for infinite dimensional systems.

Before we show the proof of Nielsen's theorem of $\epsilon$-convertibility in infinite-dimensional systems, we first show that the necessary part of the original proof of Nielsen's theorem 7 ] can be directly extended to infinite-dimensional systems by means of the Lo-Popescu and Uhlmann theorems we have already proven in the last section.

Lemma 2 The necessary condition for convertibility of an infinite-dimensional state $|\Psi\rangle$ to another state $|\Phi\rangle$ under LOCC operations is given by $\lambda \prec \mu$, where $\lambda=\{\lambda\}_{i=1}^{\infty}$ and $\mu=\{\mu\}_{i=1}^{\infty}$ are the sequences of Schmidt coefficients of the states $|\Psi\rangle$ and $|\Phi\rangle$, respectively.

Proof Suppose $|\Psi\rangle$ can be converted to $|\Phi\rangle$ by LOCC, then By Lo-Popescu's theorem, $\rho_{\Phi}=p_{m} M_{m} \rho_{\Psi} M_{m}^{\dagger}$ where $\sum_{m=1}^{\infty} p_{m}=1, \rho_{\Psi}=\operatorname{tr}{ }_{B}(|\Psi\rangle\langle\Psi|)$ and $\rho_{\Phi}=\operatorname{tr}_{B}(|\Phi\rangle\langle\Phi|)$. Then, according to the same method of Nielsen's original proof, we derive $\rho_{\Psi}=\sum_{m=1}^{\infty} p_{m} U_{m} \rho_{\Phi} U_{m}^{\dagger}$, where $U_{m}$ is a partial isometry originating in the polar decomposition of $M_{m} \sqrt{\rho_{\Psi}}$. Since $\operatorname{ker} U_{m}^{\perp}=\operatorname{ker} M_{m}{\sqrt{\rho_{\Psi}}}^{\perp} \supset \overline{\operatorname{Ran} \rho_{\psi}}[\underline{5}$, by Uhlmann's theorem we get $\lambda \prec \mu$.

By means of this lemma, we can prove the necessary part of Nielsen's theorem in infinitedimensional systems. For the sufficient condition, we fully use the properties of $\epsilon$-convertibility.

\section{Proof (Theorem 1)}

Only if part: If $|\Psi\rangle$ is $\epsilon$-convertible to $|\Phi\rangle$ for any $\epsilon>0$, there exists a sequence of states $\left\{\left|\Phi_{n}^{\prime}\right\rangle\right\}_{n=1}^{\infty}$ which strongly converges to $|\Phi\rangle$ (for pure states the topology of the trace norm is stronger than the strong topology of Hilbert space). Then, from Lemma $2, \lambda \prec \mu_{n}^{\prime}$ where $\mu_{n}^{\prime}$ and $\lambda$ are the Schmidt coefficients of $\left|\Phi_{n}^{\prime}\right\rangle$ and $|\Psi\rangle$ for all $n \in \mathbb{N}$. Because Schmidt coefficients are continuous in the strong topology, $\sum_{i=1}^{n} \mu_{n, i}^{\prime} \geq \sum_{i=1}^{n} \lambda_{i}$ means $\sum_{i=1}^{n} \mu_{i} \geq \sum_{i=1}^{n} \lambda_{i}$ where $\mu_{i}$ are the Schmidt coefficients of $|\Phi\rangle$.

If part: When the Schmidt ranks (the number of non-zero Schmidt coefficients) of both $|\Psi\rangle$ and $|\Phi\rangle$ are finite, the proof is identical to the one for finite-dimensional systems. By the definition of Schmidt decomposition, we can assume $|\Psi\rangle$ and $|\Phi\rangle$ have the same Schmidt basis without loss of generality. In what follows, we divide the proof for the remaining cases into two parts: the case where $|\Psi\rangle$ has finite Schmidt rank, and the case where both of the states have infinite Schmidt ranks.

1) The case that $|\Psi\rangle$ has infinite Schmidt rank and $|\Phi\rangle$ has finite Schmidt rank:

Suppose the Schmidt rank of $|\Phi\rangle$ is given by $N$. In what follows, we assume $\epsilon$ is arbitrary, but satisfies $\epsilon<\mu_{N}$. Since for any Schmidt coefficient $\left\{\lambda_{i}\right\}_{i=1}^{\infty}$, we have $\lim _{n \rightarrow \infty} n \lambda_{n}=0$. Therefore, there exists an $N_{1}(\epsilon)$ such that $n \lambda_{n}<\epsilon / 2$ for any $n \geq N_{1}$. On the other hand, since $\sum_{i=1}^{\infty} \lambda_{i}=1$, there exists an $N_{2}(\epsilon)$ such that $\sum_{i=n}^{\infty} \lambda_{i}<\epsilon / 2$ for any $n \geq N_{1}$. Suppose $M=\max \left(N_{1}, N_{2}, N\right)$, then we define $\left\{\mu_{i}^{\prime}\right\}$ as follows:

$$
\begin{aligned}
& \text { For } 1 \leq i \leq N-1 \quad: \mu_{i}^{\prime}=\mu_{i} \\
& i=N \quad: \mu^{\prime}{ }_{N}=\mu_{N}-\left((M-N) \lambda_{M}+\sum_{n=M+1}^{\infty} \lambda_{n}\right) \\
& N+1 \leq i \leq M \quad: \mu_{i}^{\prime}=\lambda_{M}
\end{aligned}
$$




$$
M+1 \leq i \quad: \mu_{i}^{\prime}=\lambda_{i}
$$

We define $\left|\Phi^{\prime}\right\rangle$ as $\left|\Phi^{\prime}\right\rangle=\sum_{i=1}^{\infty} \sqrt{\mu_{i}}|i\rangle \otimes|i\rangle$. Then, by definition, $\lambda \prec \mu^{\prime} \prec \mu$. Moreover, we obtain

$$
\begin{aligned}
\||\Phi\rangle-\left|\Phi^{\prime}\right\rangle \|^{2} & =\left|(M-N) \lambda_{M}+\sum_{n=M+1}^{\infty} n \lambda_{n}\right|^{2} \\
& =\left|(M-N) \lambda_{M}\right|^{2}+\left|\sum_{i=M+1}^{\infty} \lambda_{i}\right|^{2} \\
& \leq \epsilon^{2} .
\end{aligned}
$$

Therefore, for any neighborhood of $|\Phi\rangle$, we can find a $\left|\Phi^{\prime}\right\rangle$ such that $|\Psi\rangle \rightarrow\left|\Phi^{\prime}\right\rangle$.

2) The case that the Schmidt ranks of $|\Psi\rangle$ and $|\Phi\rangle$ are infinity:

By easy calculation we can show that for any $\epsilon$, there exists a natural number $N_{1}(\epsilon)$ such that if $\left|\Phi^{\prime}\right\rangle=\sum_{i=1}^{\infty} \sqrt{\mu_{i}^{\prime}}|i\rangle \otimes|i\rangle$ satisfies $\mu_{i}^{\prime}=\mu, i \leq N_{1}(\epsilon)$, then $\||\Phi\rangle\left\langle\Phi|-| \Phi^{\prime}\right\rangle\left\langle\Phi^{\prime}\right| \|_{\text {tr }}<\epsilon$. Since $\sum_{i=1}^{n} \lambda_{i}<1$ and $\lim _{n \rightarrow \infty} n \lambda_{n}=0$ for any $n \in \mathbb{N}$,

$$
\lim _{N_{2} \rightarrow \infty}\left[\sum_{k=1}^{N_{2}(\epsilon)} \lambda_{k}-\left(N_{2}-N_{1}\right) \lambda_{N_{2}}\right]=1 .
$$

Thus, there exists a natural number $N_{2}(\epsilon) \geq N_{1}(\epsilon)+1$ such that

$$
\sum_{k=1}^{N_{2}(\epsilon)} \lambda_{k}-\left(N_{2}-N_{1}\right) \lambda_{\lambda_{N_{2}}} \geq \sum_{i=1}^{N_{1}(\epsilon)} \mu_{i}
$$

and

$$
\sum_{k=1}^{N_{2}(\epsilon)-1} \lambda_{k}-\left(N_{2}-N_{1}-1\right) \lambda_{N_{2}-1} \leq \sum_{i=1}^{N_{1}(\epsilon)} \mu_{i} .
$$

We examine in the two cases $N_{2}(\epsilon)=N_{1}(\epsilon)+1$ and $N_{2}(\epsilon)>N_{1}(\epsilon)+1$ separately.

a) The case that $N_{2}(\epsilon)=N_{1}(\epsilon)+1$ : The inequalities (C.3) and (C.4) guarantee $\sum_{k=1}^{N_{1}(\epsilon)} \lambda_{k}=$ $\sum_{k=1}^{N_{1}(\epsilon)} \mu_{k}$. If we define $1 \leq k \leq N_{1}(\epsilon), \mu^{\prime}{ }_{k}=\mu_{k}, k \geq N_{1}(\epsilon)+1$ and $\mu^{\prime}{ }_{k}=\lambda_{k},\left\{\mu_{i}^{\prime}\right\}_{i=1}^{\infty}$ satisfies $\sum_{i=1}^{k} \lambda_{i} \leq \sum_{i=1}^{k} \mu_{i}^{\prime} \leq \sum_{i=1}^{k} \mu_{i}$ for all $k \in \mathbb{N}$.

b) The case that $N_{2}(\epsilon)>N_{1}(\epsilon)+1$ : We define $\delta$ as

$$
\delta=\left[\sum_{i=1}^{N_{1}(\epsilon)} \lambda_{i}+\sum_{k=N_{1}(\epsilon)+1}^{N_{2}-1}\left(\lambda_{k}-\lambda_{N_{2}}\right)-\sum_{i=1}^{N_{1}(\epsilon)} \mu_{i}\right] /\left(N_{2}(\epsilon)-N_{1}(\epsilon)-1\right) .
$$

Then, since $\delta \geq 0$, we can define $\mu^{\prime}{ }_{k}$ as the following,

$$
\begin{array}{ll}
\mu^{\prime}{ }_{k}=\mu_{k} & \text { for } 1 \leq k \leq N_{1}(\epsilon), \\
\mu^{\prime}{ }_{k}=\lambda_{N_{2}(\epsilon)}+\delta & \text { for } N_{1}(\epsilon)+1 \leq k \leq N_{2}(\epsilon)-1, \\
\mu^{\prime}{ }_{k}=\lambda_{k} & \text { for } N_{2}(\epsilon) \leq k
\end{array}
$$

then we have

$$
\begin{aligned}
\sum_{i=1}^{\infty} \mu_{i}^{\prime} & =\sum_{i=1}^{N_{1}(\epsilon)} \mu_{i}+\sum_{k=N_{1}(\epsilon)+1}^{N_{2}(\epsilon)-1}\left(\lambda_{N_{2}(\epsilon)}+\delta\right)+\sum_{k=N_{2}(\epsilon)}^{\infty} \lambda_{k} \\
& =1 .
\end{aligned}
$$


Therefore, the $\left\{\mu_{i}^{\prime}\right\}_{i=1}^{\infty}$ are well defined Schmidt coefficients.

First, we show $\sum_{i=1}^{N} \mu_{i}^{\prime} \leq \sum_{i=1}^{N} \mu_{i}$ for all $N$. Since this condition is trivial for $N \leq N_{1}$ and $N_{2} \leq N$ by definition of $\left\{\mu_{i}^{\prime}\right\}_{i=0}^{\infty}$, we only need to check this condition for $N_{1}+1 \leq N \leq N_{2}-1$. Suppose there exists an $N^{\prime}$ such that $N_{1}+1 \leq N^{\prime} \leq N_{2}-1$ and $\sum_{i=N_{1}+1}^{N^{\prime}} \mu_{i}^{\prime}>\sum_{i=N_{1}+1}^{N^{\prime}} \mu_{i}$. Then, since $\mu_{i}^{\prime}=\lambda_{N_{2}}+\delta$ for all $N_{1}+1 \leq i \leq N_{2}-1$ and $\mu_{i} \geq \mu_{i+1}$, we can easily see $\mu_{N^{\prime}}^{\prime}>\mu_{N^{\prime}}$. That is, $\sum_{i=N_{1}+1}^{N} \mu_{i}^{\prime}>\sum_{i=N_{1}+1}^{N} \mu_{i}$ for all $N^{\prime} \leq N \leq N_{2}-1$, and we can conclude $\sum_{i=N_{1}+1}^{N_{2}-1} \mu_{i}^{\prime}>\sum_{i=N_{1}}^{N_{2}-1} \mu_{i}$ which is a contradiction. Therefore, for all $N_{1}+1 \leq N \leq N_{2}-1$, $\sum_{i=N_{1}+1}^{N} \mu_{i}^{\prime} \leq \sum_{i=N_{1}}^{N} \mu_{i}$.

Second, we show $\sum_{i=1}^{N} \lambda_{i} \leq \sum_{i=1}^{N} \mu_{i}^{\prime}$ for all $N$. Since this condition is trivial for $N \leq N_{1}$ and $N_{2} \leq N$, we only check for $N_{1}+1 \leq N \leq N_{2}-1$. In this case, we calculate

$$
\begin{aligned}
\sum_{k=1}^{N} \mu_{k}{ }_{k}-\sum_{k=1}^{N} \lambda_{k}= & \sum_{k=1}^{N_{1}} \mu_{k}+\sum_{k=N_{1}+1}^{N}\left(\lambda_{N_{2}}+\delta\right)-\sum_{k=1}^{N} \lambda_{k} \\
= & \frac{N_{2}-N-1}{N_{2}-N_{1}-1}\left[\left(\sum_{k=1}^{N_{1}} \mu_{k}-\sum_{k=1}^{N_{1}} \lambda_{k}\right)-\sum_{k=N_{1}+1}^{N} \lambda_{k}\right] \\
& +\frac{N-N_{1}}{N_{2}-N_{1}-1} \sum_{k=N+1}^{N_{2}-1} \lambda_{k} .
\end{aligned}
$$

From Eq. (C.4), we obtain

$$
\sum_{k=1}^{N_{1}} \lambda_{k}+\sum_{k=N_{1}+1}^{N_{2}-2}\left(\lambda_{k}-\lambda_{N_{2}}\right) \leq \sum_{k=1}^{N_{1}} \mu_{k} .
$$

Thus, for the Eq.(C.7) may be bounded by

$$
\begin{aligned}
& \sum_{k=1}^{N} \mu_{k}^{\prime}-\sum_{k=1}^{N} \lambda_{k} \\
\geq & \frac{N_{2}-N-1}{N_{2}-N_{1}-1}\left[\sum_{k=N_{1}+1}^{N_{2}-2}\left(\lambda_{k}-\lambda_{N_{2}-1}\right)-\sum_{k=N_{1}+1}^{N} \lambda_{k}\right]+\frac{N-N_{1}}{N_{2}-N_{1}-1} \sum_{k=N+1}^{N_{2}-1} \lambda_{k} . \\
= & \sum_{k=N+1}^{N_{2}-2} \lambda_{k}-\left(N_{2}-N-2\right) \lambda_{N_{2}-1} \\
\geq & 0 .
\end{aligned}
$$

Thus, for $N_{1}+1 \leq N \leq N_{2}-1$, we have $\sum_{k=1}^{N} \lambda_{k} \leq \sum_{k=1}^{N} \mu^{\prime}{ }_{k}$.

Finally, for any natural number $N$, we obtain

$$
\sum_{k=1}^{N} \lambda_{k} \leq \sum_{k=1}^{N} \mu^{\prime}{ }_{k} \leq \sum_{k=1}^{N} \mu_{k} .
$$

We define $\left|\Phi^{\prime}\right\rangle$ by using Schmidt coefficients $\left\{\mu_{i}^{\prime}\right\}_{i=1}^{\infty}$. Then, since $\mu_{k}^{\prime}=\lambda_{k}$ for all $k \geq N_{2}(\epsilon)$, we can convert $|\Psi\rangle$ to $\left|\Phi^{\prime}\right\rangle$ by means of the LOCC operation which is derived by the original (finite dimensional) Nielsen's theorem [7. Moreover, since $\mu_{k}^{\prime}=\mu_{k}$ for all $k \leq N_{1}(\epsilon),\left|\Phi^{\prime}\right\rangle$ 
satisfies $\|\left|\Phi^{\prime}\right\rangle\left\langle\Phi^{\prime}|-| \Phi\right\rangle\langle\Phi| \|_{\text {tr }}<\epsilon$. Therefore, for any neighborhood of $|\Phi\rangle$, we can find a $\left|\Phi^{\prime}\right\rangle$ such that $|\Psi\rangle \rightarrow\left|\Phi^{\prime}\right\rangle$, where $\left|\Phi^{\prime}\right\rangle$ is defined by using Schmidt coefficients $\left\{\mu_{i}^{\prime}\right\}_{i=1}^{\infty}$.

\section{Appendix D Proof of Theorem 2}

In this appendix, based on Appendix A, B and C, we prove Vidal's 8 , theorem infinite dimensional systems.

Proof If part: The proof of Vidal's theorem in [19] is most suitable to extend this part to infinite-dimensional systems. Suppose $\lambda \prec^{\omega} p \mu$, then $\left\{\nu_{i}\right\}_{i=1}^{\infty}$ defined by the condition $\nu_{1}=1-p\left(1-\mu_{1}\right)$ and $\nu_{i}=p \mu_{i}$ for $i \neq 1$ satisfies the conditions $\lambda \prec \nu$ and $\nu \leq \mu$. If we define the state $|\Omega\rangle$ as the state whose Schmidt coefficients are $\left\{\nu_{i}\right\}$ and whose Schmidt basis is same as $|\Phi\rangle$ 's, then, by Nielsen's theorem in infinite-dimensional systems, for any small $\epsilon>0,|\Psi\rangle$ can be transformed to $\left|\Omega^{\prime}\right\rangle$ by LOCC with certainty, where $\|\left|\Omega^{\prime}\right\rangle\left\langle\Omega^{\prime}|-| \Omega\right\rangle\langle\Omega| \| \leq \epsilon$. Then, $\left|\Omega^{\prime}\right\rangle$ can also be transformed to $\left|\Phi^{\prime}\right\rangle$ by the local measurement $E=\sum_{i=1}^{\infty} \sqrt{\frac{\nu_{i}}{\mu_{i}}}|i\rangle\langle i|$ with probability $p$, where $\|\left|\Phi^{\prime}\right\rangle\left\langle\Phi^{\prime}|-| \Phi\right\rangle\langle\Phi| \|_{\text {tr }} \leq \epsilon$.

Only If part: At first, since the first half of theorem 2 of 8 is directly extended to infinitedimensional systems, Vidal's monotone $E_{n}(|\Psi\rangle)=\sum_{i=n}^{\infty} \lambda_{i}$ is a monotonic function of LOCC in mean value. In other words, if $|\Psi\rangle$ can be transformed to $\left|\Phi_{i}\right\rangle$ with probability $p_{i}$ in LOCC, then $E_{n}(|\Psi\rangle) \geq \sum_{i=1}^{\infty} p_{i} E_{n}\left(\left|\Phi_{i}\right\rangle\right)$.

If for a set of $|\Psi\rangle$ and $|\Phi\rangle$, and any $\epsilon_{1}>0$, there exists a $\left|\Phi^{\prime}\right\rangle$ such that $\|\left|\Phi^{\prime}\right\rangle\left\langle\Phi^{\prime}\right|-$ $|\Phi\rangle\langle\Phi| \|_{\text {tr }}<\epsilon_{1}$ and $|\Psi\rangle$ can be converted to $\left|\Phi^{\prime}\right\rangle$ by some SLOCC with probability $p^{\prime}$ which satisfies $p^{\prime} \geq p$ and $\lambda \prec^{\omega} p \mu$ is not true, that is for some $k_{1} E_{k_{1}}(|\Psi\rangle)<p E_{k_{1}}(|\Phi\rangle)$. Then, there exists a sequence $\left|\Phi_{n}\right\rangle$ and $p_{n}$ which satisfies $\lim _{n \rightarrow \infty}\left|\Phi_{n}\right\rangle=|\Phi\rangle$ and $p_{n} \geq p$ for all $n \in \mathbb{N}$. Moreover, there also exists a sequence of SLOCC operations which transforms $|\Psi\rangle$ to $\left|\Phi_{n}\right\rangle$. Then, by monotonicity of $E_{k}(|\Psi\rangle)$ we have $E_{k}(|\Psi\rangle) \geq p_{n} E_{k}\left(\left|\Phi_{n}\right\rangle\right)$ for all $k \in \mathbb{N}$. Since $1-E_{n}(|\Psi\rangle)$ is finite sum of eigenvalues of the reduced density operator, $E_{n}(|\Psi\rangle)$ is continuous in the whole Hilbert space and for all $n$. Thus, by taking the limit of the inequality, we have $E_{k}(|\Psi\rangle) \geq p E_{k}(|\Phi\rangle)$ for all $k \in \mathbb{N}$. This is a contradiction. 